\title{
Bürokratischer (Un-)Glücksfall
}

\author{
BEHANDELN OHNE REZEPT ALS SEKTORALER HEILPRAKTIKER Seit August 2009 schlagen \\ sich Physiotherapeuten und Ämter mit den Auswirkungen des leichtfertig gesprochenen \\ Urteils zum sektoralen Heilpraktiker herum. Bei dem Versuch, den Weg zum Behandeln \\ ohne Rezept zu beschreiben, stießen wir auf Unklarheiten - oft regierte das Chaos.
}

Stellen wir uns vor, Physiotherapeutin Hannah möchte den sektoralen Heilpraktiker im Bereich der Physiotherapie (sHP) machen und sucht nach Informationen. Ihre Ausgangsfrage: An wen muss ich mich wenden?

In jedem Bundesland ist ein anderes Amt für den neuen Bereich „sektoraler Heilpraktiker“ zuständig. Daher ist es ratsam, sich zunächst beim Landratsamt des ersten Wohnsitzes zu wenden, um dort zu erfahren, welches Amt für die Prüfungsanmeldung zuständig ist. Das kann unter anderem das Gesundheits- oder das Ordnungsamt sein.

Grundsätzlich soll man nach dem Urteil vom Bundesverwaltungsgericht in jedem Bundesland die Prüfung zum sHP ablegen können. Die entscheidende Frage lautet allerdings: Wann begannen die einzelnen Bundesländer beziehungsweise deren Gesundheitsämter mit der Umsetzung des Urteils? Manche Länder handelten rasch. In Bayern zum Beispiel fanden im Frühjahr 2010 erste Prüfungen statt. Auch in Nordrhein-Westfalen, Hessen, Berlin und Baden-Württemberg ist eine Überprüfung möglich. In Niedersachsen sollen Physiotherapeuten den sHP erhalten, wenn sie von einem fachkundigen Arzt 30 Stunden Unterricht im Fach Differenzialdiagnostik erhalten haben und dies bestätigt bekommen. In anderen Bundesländern ist die Prüfungsausarbeitung noch nicht abgeschlossen. In Sachsen ist nicht absehbar, wann es entsprechende Prüfungsrichtlinien gibt - interessierte Physiotherapeuten sollen sich frühestens 2011 melden. Physiotherapeuten in Sachsen-Anhalt sollen die Prüfung in einem anderen Bundesland ablegen.

Gibt es Zulassungsvoraussetzungen für die Prüfung?

Selbstverständlich muss man für die Prüfung zum sHP im Bereich der Physiotherapie eine abgeschlossene Berufsausbildung zum Physio- therapeuten vorweisen. Des Weiteren gelten dieselben Zulassungsvoraussetzungen wie für den allgemeinen Heilpraktiker (॰ Kasten „Zulassungsbeschränkungen“).

Wie werde ich geprüft? Wer prüft mich und was sind die Prüfungsthemen?

Da die Prüfungsdurchführung Ländersache ist, bietet jedes Bundesland eine andere Prüfung an - vorausgesetzt die zuständigen Gesundheitsämter sind schon so weit. In manchen Bundesländern wird nur mündlich geprüft, in anderen schriftlich und mündlich. In Bayern muss der Prüfling zum Beispiel zuerst eine schriftliche Prüfung ablegen, die aus 28 MultipleChoice-Fragen besteht. Innerhalb von 55 Minuten muss er mindestens 21 Fragen richtig beantworten, damit er etwa drei Wochen später zur 30-minütigen mündlichen Prüfung darf. In Baden-Württemberg wird man 30 Minuten abgefragt. In Hessen besteht die mündliche Prüfung aus drei Fragen.

\section{Jedes Bundesland hat seine eigenen Regeln.}

Einer der Prüfer muss die Prüfung selbst einmal abgelegt haben. Bisher war stellvertretend immer ein allgemeiner Heilpraktiker anwesend. Wer sonst noch prüft und vor allem welcher Facharzt anwesend ist, variiert von Prüfung zu Prüfung. Von den prüfenden Amtsärzten hängen die abgefragten Themen ab. Ist der prüfende Arzt ein Orthopäde, wird orthopädisches Wissen verlangt, während ein Chirurg eher auf sein Gebiet Wert legt. Daher ist prinzipiell mit allen Krankheitsbildern zu rechnen.

Außer der Krankheitslehre sollte man sich mit der Berufs- und Gesetzeskunde der Heilpraktiker auskennen. Da ein sHP entscheiden muss, wann seine eigene Arbeit aufhört und wann die des Arztes beginnt (sogenannter Arztvorbehalt), spielt die Differenzialdiagnostik bei der Prüfung eine große Rolle. Vor allem in Bezug auf bösartige Erkrankungen, Volksund Infektionskrankheiten, die im Infektionsschutzgesetz genannt werden, sollte man differenzialdiagnostisches Wissen beweisen.

Im Urteil des Bundesverwaltungsgerichts heißt es, die Prüfung müsse sich auf solche Kenntnisse beschränken, die zur eigenverantwortlichen Anwendung von Physiotherapie erforderlich und nicht bereits durch die Berufsausbildung vermittelt worden seien. Ist es nicht paradox, eine Prüfung auf diese Weise gestalten zu wollen?

Das ist der Grund, warum es so schwer ist, eine Prüfung zu gestalten, die rechtlich einwandfrei ist. Einerseits sollen die Prüfungen keine billige Zulassung für den SHP sein, denn es muss gewährleistet sein, dass keine Gefahr vom sHP ausgeht. Andererseits will man sich aber auch keine Klagewelle wegen unzulässiger Fragen aufhalsen. Denn die Prüfung darf von den Physiotherapeuten nichts verlangen, was ihnen nicht zugemutet werden darf.

Ganz besonders schwierig ist es, in der Prüfung Themengebiete auszuklammern, „die zur eigenverantwortlichen Anwendung von Physiotherapie“ nicht gebraucht werden. Hier verschwimmen die Grenzen zur Differenzialdiagnostik. Ein Prüfling darf beispielsweise nicht nach genauen Blutwerten gefragt werden, da er dies für seine Berufsausübung nicht benötigt. Wollen die Prüfer aber das differenzialdiagnostische Wissen testen, sind Fragen zu den Blutwerten nahezu unumgänglich.

Wie hoch ist die Durchfallquote? Kann ich die Prüfung wiederholen? Wie kann ich mich optimal vorbereiten? 


\section{Was kostet mich die Prüfung?}

Da nicht jedes Bundesland gleich prüft, sind auch die Gebühren nicht einheitlich.

In Bayern kostet die schriftliche Prüfung beispielsweise zwischen 200,- und 250,- $€$, die mündliche noch einmal 200,- $€$. Für die Urkunde muss man zwischen 30,- und 40,- € zahlen.

Als weiteres Beispiel kann Baden-Württemberg genannt werden: Hier zahlt man für die mündliche Prüfung etwa 260,- $€$. Dafür muss man für die Urkunde tiefer in die Tasche greifen - sie kostet $160,-€$.

\section{Was darf ich als sHP alles behandeln, was ich vorher als Physiotherapeut nicht durfte? \\ Im Gegensatz zum Physiotherapeuten hat der sHP das Recht zum Erstkontakt - das heißt, er darf auch Patienten ohne ärztliches Rezept befunden, diagnostizieren und behandeln. $\mathrm{Er}$ darf alle Krankheitsbilder annehmen, muss aber im Gebiet der Physiotherapie bleiben - sowohl beim Befund als auch in der Therapie, denn „eine heilkundliche Betätigung außer- halb der Physiotherapie ist unzulässig“ und so- mit strafbar. Der HP muss seine Grenzen ganz genau kennen, sodass er den Patienten gege- benenfalls zum Arzt überweist. Zu diesen Grenzen gehören unter anderem bestimmte Tätigkeitsverbote, die dem Heilpraktikerge- setz und dem Heilmittelwerbegesetz ent- stammen (॰ Kasten „Zusatzinfos“).}

\section{Ein sektoraler Heilpraktiker muss seine Grenzen kennen.}

Reicht der sHP in der Physiotherapie aus, damit ich auch osteopathisch tätig sein darf? Hier sind die Länder bisher zu keiner Regelung vorgedrungen.

\section{Wie ist die Rechtslage? Was ist mit der Prü- fung zum sHP anders als vorher?}

Bisher herrschte eine rechtliche Grauzone, wenn es um die physiotherapeutische Behandlung eines Patienten ohne ärztliches Rezept ging. Die rechtliche Sicherheit des Physiotherapeuten war diesbezüglich nie eindeutig geklärt, doch mit dem Urteil des Bundesverwaltungsgerichts ist die Rechtslage klar: Wer Patienten auf Selbstzahlerbasis ohne Rezept physiotherapeutisch behandeln möchte, braucht den SHP - andernfalls macht er sich strafbar. Ohne sektoralen Heilpraktiker sind Physiotherapeuten weisungsgebunden und benötigen eine ärztliche Verordnung, welche ihre therapeutische Intervention bestimmt.

Auch in Bezug auf die haftende Versicherung ist Klarheit eingetreten. Der sHP sollte seine Teilheilpraktikererlaubnis der Berufshaftpflichtversicherung vorlegen, um die Versicherungspolice auf eine Tätigkeit ohne ärztliche Verordnung zu erweitern.

\section{Darf ich mit meinem neu erworbenen Titel werben?}

Physiotherapeuten dürfen mit ihrem neuen Titel werben. Allerdings müssen sie die Vorschriften des Heilmittelwerbegesetzes beachten. Teilweise finden sich diese in den Tätigkeitsverboten ( $\odot$ Kasten „Zusatzinfos“). Des Weiteren muss im Wesentlichen auf vier Wettbewerbsstrategien verzichtet werden:

$>$ Werbung mit Abbildungen, die den Therapeuten beispielsweise in Berufskleidung oder bei der beruflichen Betätigung zeigen,

$>$ Werbung, die Zuwendungen versprechen oder gewähren, zum Beispiel „Zahlen Sie 6 Massagen und erhalten 7“, oder Weihnachtspräsente verschenken,

$>$ Werbung mit fremd- oder fachsprachlichen Begriffen wie Osteopathie oder TCM in Zeitungsannoncen oder Telefonbucheinträgen,

$>$ Werbung, die gleichzeitig die Krankheit und das therapeutische Mittel nennt, zum Beispiel: „Haben Sie ein Phlebödem? Dann kommen Sie zur Manuellen Lymphdrainage zu mir.“

\section{Ersetzt der sHP den Direktzugang?}

Ein Physiotherapeut, der die Prüfung zum sHP abgelegt hat, darf Patienten ohne ärztliche Verordnung behandeln, doch die Patienten bezahlen die Therapie selbst. Die Berufsverbände setzen sich weiter für den Direktzugang ein, damit eine Versorgung innerhalb des Krankenkassensystems bestehen bleibt. Manche sehen im sHP allenfalls einen Schritt in die richtige Richtung, aber keinen Ersatz für das eigentliche Ziel - den Direktzugang.

\section{Lohnt sich der sHP für mich?}

Die Leistungen der Krankenkassen werden immer weiter zurückgehen, sodass selbst zahlende Patienten für den Physiotherapeuten mehr und mehr an Bedeutung gewinnen. Um dabei rechtlich abgesichert behandeln zu können, ist der sHP in der Physiotherapie notwendig.
Doch bevor man entscheidet, ob sich ein sHP lohnt, sollte man sich folgende Frage stellen: Wie viele selbst zahlende Patienten erwarte ich in meiner Praxis?

Auch Hannah muss sich zunächst überlegen, ob ihre Klientel die „neuen“ Leistungen in ihrer Praxis in Anspruch nehmen würde, damit sie von einem sHP profitiert. Vieles spricht dafür, doch auf der anderen Seite herrscht noch ein zu großes Chaos. Hannah wartet vielleicht auch erst einmal ab...

Kathrin Bauer

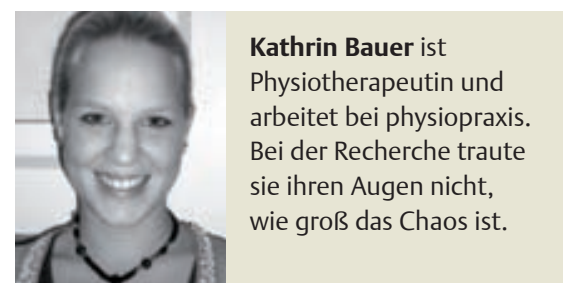

ZUSATZINFOS

\section{Beispielfragen und Tätigkeitsverbote}

Im Internet finden Sie unter www. thieme-connect.de/ejournals/toc/ physiopraxis alle 28 Prüfungsfragen einer schriftlichen Prüfung zum sHP in Bayern. Außerdem finden Sie dort die geltenden Tätigkeitsverbote.

\section{DANIKSAGUNG}

Wir bedanken uns bei unseren Interviewpartnern für die freundliche Unterstützung und die vielen hilfreichen Informationen:

Dr. jur. Ernst Boxberg (Rechtsanwalt/Fachanwalt für Medizinrecht) Karlheinz Hagmeyer (selbstständiger Physiotherapeut und sektoraler Heilpraktiker in der Physiotherapie) Eva Maria Reichart (selbstständige Physiotherapeutin und allgemeine Heilpraktikerin im Gesundheitshaus Reichart, Mitglied im Vorstand/ Schatzmeisterin des ZVK LV Bayern) Rolf Schneider (Medizinpädagoge und allgemeiner Heilpraktiker, Gründer der staatlichen Naturheilkundeschulen Rolf Schneider Seminare) Peter Stojanoff (Landesvorsitzender der VPT Landesgruppe BW) 